\title{
Accurate and efficient computation of the Green's tensor for stratified media
}

\author{
Michael Paulus, ${ }^{1,2}$ Phillipe Gay-Balmaz, ${ }^{1}$ and Olivier J. F. Martin ${ }^{1, *}$ \\ ${ }^{1}$ Electromagnetic Fields and Microwave Electronics Laboratory, Swiss Federal Institute of Technology, ETH-Zentrum ETZ, \\ CH-8092 Zurich, Switzerland \\ ${ }^{2}$ IBM Research Division, Zurich Research Laboratory, CH-8803 Rüschlikon, Switzerland
}

(Received 28 April 2000)

\begin{abstract}
We present a technique for the computation of the Green's tensor in three-dimensional stratified media composed of an arbitrary number of layers with different permittivities and permeabilities (including metals with a complex permittivity). The practical implementation of this technique is discussed in detail. In particular, we show how to efficiently handle the singularities occurring in Sommerfeld integrals, by deforming the integration path in the complex plane. Examples assess the accuracy of this approach and illustrate the physical properties of the Green's tensor, which represents the field radiated by three orthogonal dipoles embedded in the multilayered medium.
\end{abstract}

PACS number(s): 42.25.Bs, 42.25.Fx, 42.25.Gy, 42.68.Mj

\section{INTRODUCTION}

The Green's tensor $\mathbf{G}\left(\mathbf{r}, \mathbf{r}^{\prime}\right)$ represents the electric field radiated at position $\mathbf{r}$ by three orthogonal dipoles located at $\mathbf{r}^{\prime}$. For an infinite homogeneous background, this dyadic can be computed analytically $[1,2]$. The situation is much more complex in a stratified medium, where the Green's tensor must take into account all the reflections and refractions that occur at the different interfaces.

The simplest geometry of interest here is that of a dipolar source above a surface. This problem was first treated by Sommerfeld [3] and then extensively studied in connection with antennae radiation [4-7].

In this context of a single interface, the intrinsic properties of the Green's tensor were also used to investigate the variation of spontaneous emission for a dipolar transition in the presence of a surface [8-11]. Similar properties were also utilized to determine the polariton spectrum in semiconductor superlattices [12].

The Green's tensor plays a key role in scattering calculations. As a matter of fact, when a scatterer is discretized into small enough elements, the response of each element to an external field is essentially dipolar [13] and the Green's tensor gives the interaction between these discretized elements. This technique, known also as the coupled dipole approximation, has been successfully applied to many scattering problems in infinite homogeneous backgrounds [14-16]. Using the Green's tensor associated with a surface, it was also used to investigate scattering on a surface [17-21].

In this paper we present an efficient technique for the accurate computation of the Green's tensor for arbitrarily layered media (i.e., not limited to a single interface). The formalism is derived in Sec. II. It leads to so-called Sommerfeld integrals that must be performed numerically in the complex plane. Our numerical implementation is described in Sec. III. In Sec. IV we illustrate the physical properties of the Green's tensor and summarize our work in Sec. V. Ap-

\footnotetext{
*Correspondence author. Email address: martin@ifh.ee.ethz.ch
}

pendixes A and B give the detailed formulas for the practical implementation of this technique.

\section{FORMALISM}

The Green's tensor $\mathbf{G}\left(\mathbf{r}, \mathbf{r}^{\prime}\right)$ for an arbitrary scattering system described by the dielectric permittivity $\epsilon(\mathbf{r})$ and the magnetic permeability $\mu(\mathbf{r})$ is the solution of the vector wave equation with a point source term [1]:

$$
\begin{aligned}
\boldsymbol{\nabla} \times \mu^{-1}(\mathbf{r}) \boldsymbol{\nabla} \times \mathbf{G}\left(\mathbf{r}, \mathbf{r}^{\prime}\right)-k_{0}^{2} \epsilon(\mathbf{r}) \boldsymbol{\nabla} \times \mathbf{G}\left(\mathbf{r}, \mathbf{r}^{\prime}\right) \\
=\mu^{-1}(\mathbf{r}) \mathbf{1} \delta\left(\mathbf{r}-\mathbf{r}^{\prime}\right),
\end{aligned}
$$

where $k_{0}^{2}=\omega^{2} \epsilon_{0} \mu_{0}$ is the vacuum wave number [throughout the paper we assume time harmonic fields with an $\exp (-i \omega t)$ dependence].

In this paper we specifically consider planar stratified systems, where each layer $l=1, \ldots, N$ can be characterized by the permittivity $\epsilon_{l}$ and the permeability $\mu_{l}$ as illustrated in Fig. 1. For convenience, we chose our coordinate system such that the layers are parallel to the $x y$ plane.

For the derivation of $\mathbf{G}\left(\mathbf{r}, \mathbf{r}^{\prime}\right)$ associated with a stratified background, we start with the expression for the free-space Green's tensor $\mathbf{G}_{H}\left(\mathbf{r}, \mathbf{r}^{\prime}\right)$ of a homogeneous system $\epsilon_{B}$ $=\epsilon_{0} \epsilon_{r}, \mu_{B}=\mu_{0} \mu_{r}$. It is given by [16]

$$
\begin{aligned}
\mathbf{G}_{H}\left(\mathbf{r}, \mathbf{r}^{\prime}\right)= & \left(\mathbf{1}+\frac{\boldsymbol{\nabla} \boldsymbol{\nabla}}{k_{B}^{2}}\right) \frac{\exp \left(i k_{B} R\right)}{4 \pi R} \\
= & \left(\mathbf{1}+\frac{i k_{B} R-1}{k_{B}^{2} R^{2}} \mathbf{1}+\frac{3-3 i k_{B} R-k_{B}^{2} R^{2}}{k_{B}^{2} R^{4}} \mathbf{R} \mathbf{R}\right) \\
& \times \frac{\exp \left(i k_{B} R\right)}{4 \pi R},
\end{aligned}
$$

where $R=|\mathbf{R}|=\left|\mathbf{r}-\mathbf{r}^{\prime}\right|$ is the relative distance and $k_{B}^{2}$ $=\omega^{2} \epsilon_{B} \mu_{B}$ corresponds to the wave number in the background medium. 


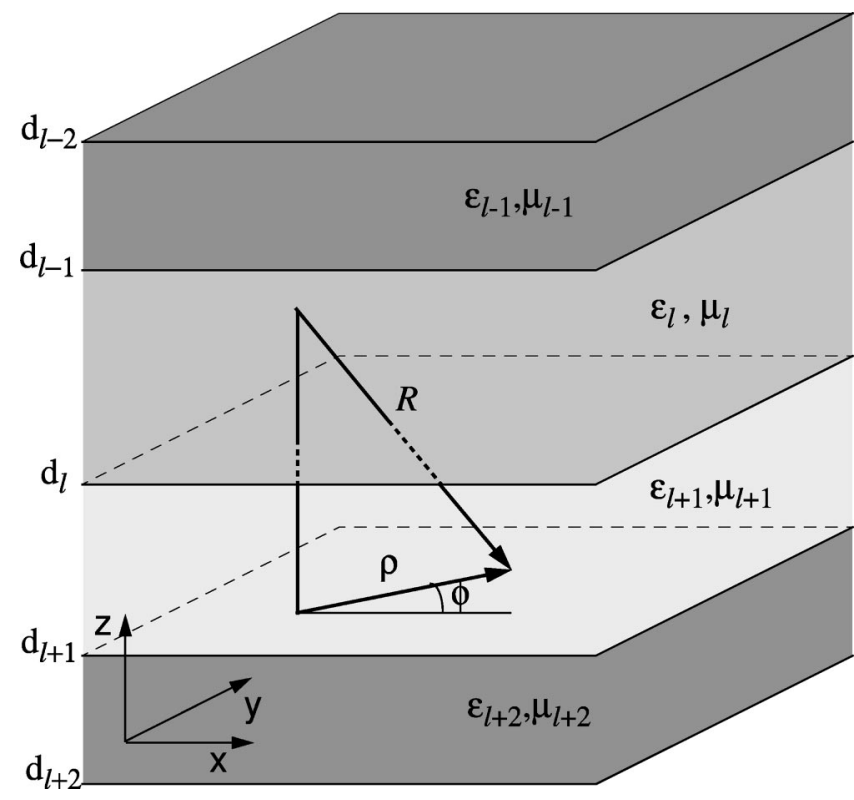

FIG. 1. Stratified medium consisting of $N$ layers with $\left(\epsilon_{1}, \mu_{1}\right), \ldots,\left(\epsilon_{N}, \mu_{N}\right)$ separated by interfaces at $z$ $=d_{1}, \ldots, d_{N-1}$. The vector $\mathbf{R}=\mathbf{r}-\mathbf{r}^{\prime}$ defines the relative distance between $\mathbf{r}$ and $\mathbf{r}^{\prime}$ and $\boldsymbol{\rho}=\left(x-x^{\prime}, y-y^{\prime}\right)=(\rho \cos \phi, \rho \sin \phi)$ is the projection of $\mathbf{R}$ onto the $x y$ plane.

To deduce $\mathbf{G}\left(\mathbf{r}, \mathbf{r}^{\prime}\right)$ for a stratified background, it is more convenient to use an integral representation of $\mathbf{G}_{H}\left(\mathbf{r}, \mathbf{r}^{\prime}\right)$. Fourier transforming Eq. (2) leads to

$$
\mathbf{G}_{H}\left(\mathbf{r}, \mathbf{r}^{\prime}\right)=\frac{1}{8 \pi^{3} k_{B}^{2}} \iiint d \mathbf{k}\left(\frac{1 k_{B}^{2}-\mathbf{k k}}{k^{2}-k_{B}^{2}}\right) \exp (i \mathbf{k} \cdot \mathbf{R})
$$

Since we assume that the layers, which will be added later, are perpendicular to the $z$ axis, we first perform the integration over $k_{z}$ using calculus of residues. Hence, we must ensure that the integrand vanishes for $k_{z} \rightarrow \infty$ and rewrite Eq. (4) as

$$
\begin{aligned}
\mathbf{G}_{H}\left(\mathbf{r}, \mathbf{r}^{\prime}\right)= & \frac{1}{8 \pi^{3} k_{B}^{2}} \iiint d \mathbf{k}\left(\frac{\mathbf{1} k_{B}^{2}-\mathbf{k} \mathbf{k}}{k^{2}-k_{B}^{2}}+\hat{\mathbf{z}}\right) \exp (i \mathbf{k} \cdot \mathbf{R}) \\
& -\frac{\hat{\mathbf{z}} \mathbf{z}}{8 \pi^{3} k_{B}^{2}} \iiint d \mathbf{k} \exp (i \mathbf{k} \cdot \mathbf{R}) \\
= & \frac{i}{8 \pi^{2} k_{B}^{2}} \iint d k_{x} d k_{y}\left(\frac{\mathbf{1} k_{B}^{2}-\mathbf{k}_{B}}{k_{B z}}\right) \\
& \times \exp \left(i \mathbf{k}_{B} \cdot \mathbf{R}\right)-\frac{\hat{\mathbf{z}}}{k_{B}^{2}} \delta(\mathbf{R}),
\end{aligned}
$$

where $k_{B z}=\left(k_{B}^{2}-k_{x}^{2}-k_{y}^{2}\right)^{1 / 2}$ is the $z$ component of the wave vector and

$$
\mathbf{k}_{B}\left(k_{B z}\right)=\left\{\begin{array}{ll}
k_{x} \hat{\mathbf{x}}+k_{y} \hat{\mathbf{y}}+k_{B z} \hat{\mathbf{z}} & \text { for } z>z^{\prime}, \\
k_{x} \hat{\mathbf{x}}+k_{y} \hat{\mathbf{y}}-k_{B z} \hat{\mathbf{z}} & \text { for } z<z^{\prime}
\end{array} .\right.
$$

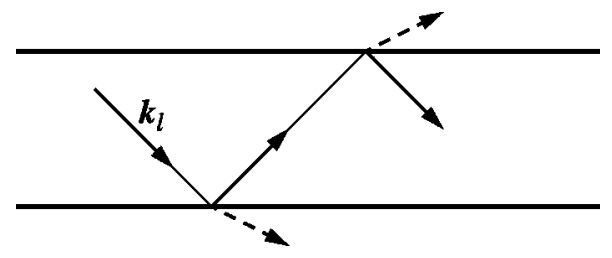

FIG. 2. The effect of the surrounding layers on a given Fourier component $\mathbf{k}_{l}$ in layer $l$ is to add an upgoing and a downgoing plane wave with similar $\mathbf{k}$ vectors.

Now that we have the plane-wave expansion of the Green's tensor for an infinite homogeneous background $\epsilon_{B}, \mu_{B}$, it is a simple matter to include additional layers. Indeed, the effect of these layers will be to add two plane waves, one propagating upward and one downward, to each Fourier component, as illustrated in Fig. 2 [note that the propagation vector $\left(k_{x}, k_{y}, k_{z}\right)$ is similar for the three plane waves, only the sign of $k_{z}$ changes]. The amplitudes of these additional components are determined by the boundary conditions at the different interfaces. Since the Green's tensor represents the electric field radiated at $\mathbf{r}$ by three orthogonal point sources at $\mathbf{r}^{\prime}$, the boundary conditions depend on the polarization of the corresponding Fourier component. It is therefore advantageous to introduce a new orthonormal system $\hat{\mathbf{k}}\left(k_{B z}\right), \hat{\mathbf{l}}\left(k_{B z}\right)$, and $\hat{\mathbf{m}}\left(k_{B z}\right)[22]$,

$$
\begin{gathered}
\hat{\mathbf{k}}\left(k_{B z}\right)=\frac{\mathbf{k}_{B}\left(k_{B z}\right)}{k_{B}}, \\
\hat{\mathbf{l}}\left(k_{B z}\right)=\frac{\hat{\mathbf{k}}\left(k_{B z}\right) \times \hat{\mathbf{z}}}{\left|\hat{\mathbf{k}}\left(k_{B z}\right) \times \hat{\mathbf{z}}\right|}, \\
\hat{\mathbf{m}}\left(k_{B z}\right)=\hat{\mathbf{k}}\left(k_{B z}\right) \times \hat{\mathbf{l}}\left(k_{B z}\right) .
\end{gathered}
$$

Equivalently, another orthonormal system is formed by $\hat{\mathbf{k}}\left(-k_{B z}\right)$, $\hat{\mathbf{l}}\left(-k_{B z}\right)$, and $\hat{\mathbf{m}}\left(-k_{B z}\right)$. Remark that $\hat{\mathbf{l}}$ is perpendicular to the plane defined by $\hat{\mathbf{k}}$ and $\hat{\mathbf{z}}$, whereas $\hat{\mathbf{m}}$ lies within this plane. For a given $k_{B}$, the electric-field component parallel to $\hat{\mathbf{l}}$ corresponds, therefore, to $s$ polarization and that parallel to $\hat{\mathbf{m}}$ corresponds to $p$ polarization. Using the fact that $\hat{\mathbf{k}} \hat{\mathbf{k}}+\hat{\mathbf{l}} \mathbf{\mathbf { l }}+\hat{\mathbf{m}} \hat{\mathbf{m}}=\mathbf{1}$, Eq. (5) can be rewritten as

$$
\begin{aligned}
\mathbf{G}_{H}\left(\mathbf{r}, \mathbf{r}^{\prime}\right)= & -\frac{\hat{\mathbf{z}} \mathbf{z}}{k_{B}^{2}} \delta(\mathbf{R})+\frac{i}{8 \pi^{2}} \iint d k_{x} d k_{y}\left(\frac{\hat{\mathbf{l}} \mathbf{\mathbf { l }}+\hat{\mathbf{m}} \hat{\mathbf{m}}}{k_{B z}}\right) \\
& \times \exp \left(i \mathbf{k}_{B} \cdot \mathbf{R}\right) .
\end{aligned}
$$

To obtain the Green's tensor $\mathbf{G}\left(\mathbf{r}, \mathbf{r}^{\prime}\right)$ for a stratified medium, we can now superpose to the free-space Green's tensor of a homogeneous medium $\epsilon_{l}, \mu_{l}$ the additional terms by formally writing

$$
\begin{aligned}
\mathbf{G}\left(\mathbf{r}, \mathbf{r}^{\prime}\right)= & -\frac{\hat{\mathbf{z}}}{k_{l}^{2}} \delta(\mathbf{R})+\frac{i}{8 \pi^{2}} \iint d k_{x} d k_{y} \exp \left\{i \left[k_{x}\left(x-x^{\prime}\right)\right.\right. \\
& \left.\left.+k_{y}\left(y-y^{\prime}\right)\right]\right\} \frac{1}{k_{l z}}\left[\hat{\mathbf{l}} \exp \left(i k_{l z}\left|z-z^{\prime}\right|\right)\right. \\
& +\mathbf{R}^{s \uparrow} \exp \left(i k_{l z} z\right)+\mathbf{R}^{s \downarrow} \exp \left(-i k_{l z} z\right)
\end{aligned}
$$




$$
\begin{aligned}
& +\hat{\mathbf{m}} \hat{\mathbf{m}} \exp \left(i k_{l z}\left|z-z^{\prime}\right|\right)+\mathbf{R}^{p \uparrow} \exp \left(i k_{l z} z\right) \\
& \left.+\mathbf{R}^{p \downarrow} \exp \left(-i k_{l z} z\right)\right],
\end{aligned}
$$

where $k_{l}^{2}=\omega^{2} \epsilon_{l} \mu_{l}$ and $k_{l z}=\left(k_{l}^{2}-k_{x}^{2}-k_{y}^{2}\right)^{1 / 2}$. The tensors $\mathbf{R}^{s \uparrow}, \mathbf{R}^{s \downarrow}, \mathbf{R}^{p \uparrow}$, and $\mathbf{R}^{p \downarrow}$ can obviously be interpreted as generalized reflection coefficients that take into account reflections from all existing surfaces.

For the explicit calculation of $\mathbf{G}\left(\mathbf{r}, \mathbf{r}^{\prime}\right)$ it is necessary to consider separately the two cases $z>z^{\prime}$ and $z<z^{\prime}$. In Eq. (9) each component of the Green's tensor is expressed in terms of $s$ - and $p$-polarized upgoing and downgoing plane waves with amplitude coefficients $A_{l, \alpha \beta}^{s}, B_{l, \alpha \beta}^{s}, A_{l, \alpha \beta}^{p}$, and $B_{l, \alpha \beta}^{p}$ :

$$
\begin{aligned}
G_{\alpha \beta}\left(\mathbf{r}, \mathbf{r}^{\prime}\right)= & \frac{i}{8 \pi^{2}} \iint d k_{x} d k_{y} \exp \left\{i \left[k_{x}\left(x-x^{\prime}\right)\right.\right. \\
& \left.\left.+k_{y}\left(y-y^{\prime}\right)\right]\right\} \frac{1}{k_{l z}}\left\{\left[\hat{l}_{\alpha}\left( \pm k_{l z}\right) A_{l, \alpha \beta}^{s} \exp \left(i k_{l z} z\right)\right.\right. \\
& \left.+\hat{l}_{\alpha}\left(\mp k_{l z}\right) B_{l, \alpha \beta}^{s} \exp \left(-i k_{l z} z\right)\right] \hat{l}_{\beta}\left(k_{l z}\right) \\
& +\left[\hat{m}_{\alpha}\left( \pm k_{l z}\right) A_{l, \alpha \beta}^{p} \exp \left(i k_{l z} z\right)\right. \\
& \left.\left.+\hat{m}_{\alpha}\left(\mp k_{l z}\right) B_{l, \alpha \beta}^{p} \exp \left(-i k_{l z} z\right)\right] \hat{m}_{\beta}\left(k_{l z}\right)\right\}
\end{aligned}
$$

where the upper sign refers to the case $z>z^{\prime}$ and the lower sign to $z<z^{\prime}$. For simplicity, the contribution of the $\delta$ function is omitted in Eq. (10). The amplitude coefficients $A_{l, \alpha \beta}^{s}$, $B_{l, \alpha \beta}^{s}, A_{l, \alpha \beta}^{p}$, and $B_{l, \alpha \beta}^{p}$ are functions of $\left(k_{l z} ; \mathbf{r}, \mathbf{r}^{\prime}\right)$ and are derived in Appendix A.

Equation (10) can be further simplified by introducing a cylindrical coordinate system. In the $x y$ and $k_{x} k_{y}$ plane we introduce the transverse coordinate $\boldsymbol{\rho}$,

$$
\begin{gathered}
\mathbf{R}=\left(\boldsymbol{\rho}, z-z^{\prime}\right)=\left(x-x^{\prime}, y-y^{\prime}, z-z^{\prime}\right), \\
\boldsymbol{\rho}=(\rho \cos \phi, \rho \sin \phi),
\end{gathered}
$$

and the transverse wave vector $\mathbf{k}_{\rho}$,

$$
\begin{aligned}
\mathbf{k} & =\left(\mathbf{k}_{\rho}, k_{z}\right)=\left(k_{x}, k_{y}, k_{z}\right), \\
\mathbf{k}_{\rho} & =\left(k_{\rho} \cos k_{\phi}, k_{\rho} \sin k_{\phi}\right) .
\end{aligned}
$$

The integration over $k_{\phi}$ in Eq. (10) is then performed analytically with the help of Bessel functions [23]:

$$
J_{n}\left(k_{\rho} \rho\right)=\frac{i^{-n}}{2 \pi} \int_{0}^{2 \pi} d k_{\phi} \exp \left(i k_{\rho} \rho \cos k_{\phi}\right) \cos \left(n k_{\phi}\right) .
$$

Thus, only a one-dimensional integral over the radial component $k_{\rho}$ must be calculated numerically:

$$
\begin{aligned}
\mathbf{G}\left(\mathbf{r}, \mathbf{r}^{\prime}\right)= & -\frac{\hat{\mathbf{z}}}{k_{l}^{2}} \delta(\mathbf{R}) \\
& +\frac{i}{4 \pi} \int_{0}^{\infty} d k_{\rho}\left[\mathbf{f}^{s}\left(k_{\rho} ; \mathbf{r}, \mathbf{r}^{\prime}\right)+\mathbf{f}^{p}\left(k_{\rho} ; \mathbf{r}, \mathbf{r}^{\prime}\right)\right] .
\end{aligned}
$$

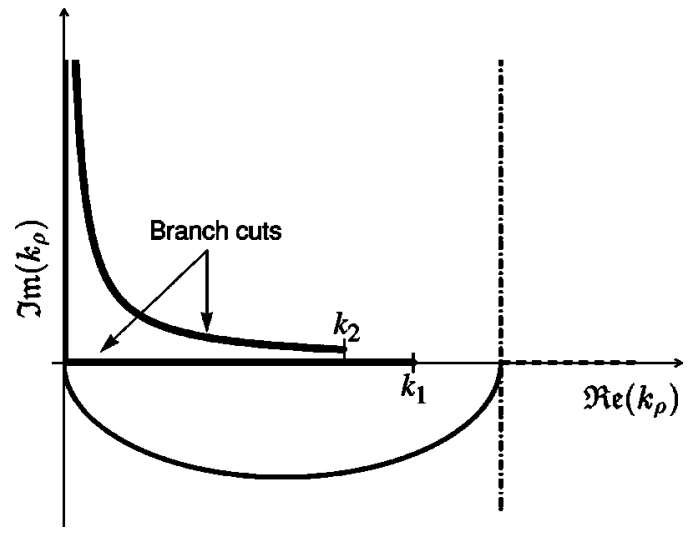

FIG. 3. Plane of integration: The singularities are surrounded by an elliptical path (solid line). At $k_{\rho}=2 k^{\text {maj }}$ the integration is resumed along the real $k_{\rho}$ axis using Bessel functions (dashed line) or parallel to the imaginary $k_{\rho}$ axis using Hankel functions (dashdotted line).

The components of the tensors $\mathbf{f}^{s}$ and $\mathbf{f}^{p}$, obtained after careful evaluation of Eq. (10), are given in Appendix B.

\section{PRACTICAL IMPLEMENTATION}

The objective of this paper being to provide a useful framework for the computation of the Green's tensor for stratified media, we discuss in this section the practical details related to the numerical evaluation of Eq. (16).

\section{A. Singularities}

In the preceding section the Green's tensor for stratified media was expressed as a set of one-dimensional semiinfinite integrals, so-called Sommerfeld integrals [Eqs. (16) and (B1)-(B18)]. A typical integrand has the form

$$
\begin{aligned}
G\left(k_{\rho} ; \mathbf{r}, \mathbf{r}^{\prime}\right)= & g\left(k_{\rho} ; \mathbf{r}, \mathbf{r}^{\prime}\right) J_{n}\left(k_{\rho} \rho\right)\left[\mathcal{A}\left(k_{\rho}, z^{\prime}\right)\right. \\
& \left.\times \exp \left(i k_{l z} z\right)+\mathcal{B}\left(k_{\rho}, z^{\prime}\right) \exp \left(-i k_{l z} z\right)\right] .
\end{aligned}
$$

An integral of this kind cannot be performed analytically, but has to be evaluated numerically. However, a straightforward implementation would fail because of the mathematically awkward behavior of the integrand. To avoid these difficulties we use Cauchy's integral theorem and deform the integration path in the complex $k_{\rho}$ plane. The objective of this section is to determine such an optimum path.

The singularities of $G\left(k_{\rho} ; \mathbf{r}, \mathbf{r}^{\prime}\right)$ can be classified in two types: branch point singularities and pole singularities. Branch point singularities are related to the $k_{l z}=\left(k_{l}^{2}\right.$ $\left.-k_{\rho}^{2}\right)^{1 / 2}$ dependence of the integrands. Since $k_{l z}$ is the square root of a complex number, it is double valued and branch cuts defined by $\operatorname{Im}\left(k_{l z}\right)=0$ intersect the plane of integration [1]. Each branch cut ends in a branch point at $k_{\rho}= \pm k_{l}$. If the medium is lossless, these singularities are located on the real $k_{\rho}$ axis, i.e., on the integration path (Fig. 3).

One could expect that a branch cut exists for each layer, i.e., for each $k_{l}$. However, it can be shown that the functional dependence of $G\left(k_{\rho} ; \mathbf{r}, \mathbf{r}^{\prime}\right)$ on $k_{l z}$ is even for all $l$ except the two outermost regions [24,25]. Therefore, branch cuts and branch points appear only for $k_{\rho}= \pm k_{1}, \pm k_{N}$. 
Since the integral runs from zero to infinity, we can further restrict our discussion to singularities with a non-negative real part.

To choose now the appropriate value for $k_{1 z}$ and $k_{N z}$, thereby ensuring that the integration is performed on the correct Riemann sheet defined by the branch cut $\operatorname{Im}\left(k_{l z}\right)=0$, we simply apply the radiation conditions: We must use the value with $\operatorname{Im}\left(k_{l z}\right)>0$ to make the integrands vanish for $z \rightarrow \pm \infty$.

The second class of singularities, pole singularities, is due to vanishing denominators of $G\left(k_{\rho} ; \mathbf{r}, \mathbf{r}^{\prime}\right)$. These poles correspond physically to modes guided by the layered structure, as will be illustrated in Sec. IV. For dielectric media, such modes have propagation vectors $k_{\rho}$ smaller than $k_{l}^{\max }$ $=\max _{l} \operatorname{Re}\left(k_{l}\right)$ [26].

For lossy materials with $\operatorname{Im}(\epsilon)>0$, it can be shown that both branch points and pole singularities are shifted into the first quadrant of the complex $k_{\rho}$ plane. However, if the losses are small, the singularities remain close enough to the $\operatorname{Re}\left(k_{\rho}\right)$ axis and can still cause serious problems.

\section{B. Numerical quadrature}

To avoid singularities, our integration path must therefore run inside the fourth quadrant in the complex $k_{\rho}$ plane. A possible choice would be to leave the real axis only in close vicinity of the singularities, e.g., by a semicircle around each singularity. This requires us to precisely determine the location of each pole, which is a tedious and time-consuming task [27].

In our implementation we use an easier way of surrounding the singularities: an elliptical path starting at $k_{\rho}=0$ with the major semiaxis $k_{\rho}^{\text {maj }}$ and the minor semiaxis $k_{\rho}^{\mathrm{min}}$ (Fig. 3). Of course, we must ensure that $k_{\rho}^{\mathrm{maj}}$ is chosen large enough to enclose all the singularities. From the discussion in the preceding section we take $2 k_{\rho}^{\mathrm{maj}}=k_{l}^{\mathrm{max}}+k_{0}$, where the vacuum wave number $k_{0}$ is added as a safety margin.

The parameter $k_{\rho}^{\min }$ is empirically chosen such that the integration path is sufficiently far away from the singularities without extending too much in negative imaginary $k_{\rho}$ direction, because the Bessel function $J_{n}\left(k_{\rho} \rho\right)$ increases then very rapidly. We have observed that $k_{\rho}^{\mathrm{min}}=10^{-3} k_{\rho}^{\mathrm{maj}}$ was a good choice for this parameter. A typical integrand is shown in Fig. 4, together with the deformed integration path.

For the remaining integration, we usually follow the real $k_{\rho}$ axis. However, when $z-z^{\prime}$ is small, the exponential damping in Eq. (17) becomes weak and the quadrature taken along the real axis converges very slowly. In that case, it is more advantageous to transform the integral using Hankel functions:

$$
\begin{aligned}
J_{n}\left(k_{\rho} \rho\right) & =\frac{1}{2}\left[H_{n}^{(1)}\left(k_{\rho} \rho\right)+H_{n}^{(2)}\left(k_{\rho} \rho\right)\right] \\
& =\frac{1}{2}\left\{H_{n}^{(1)}\left(k_{\rho} \rho\right)+\left[H_{n}^{(1)}\left(\left(k_{\rho} \rho\right)^{*}\right)\right]^{*}\right\} .
\end{aligned}
$$

Because of the asymptotic behavior of the Hankel functions for large values of $k_{\rho} \rho$,

$$
\lim _{\left|k_{\rho} \rho\right| \rightarrow \infty} H_{n}^{(1)}\left(k_{\rho} \rho\right)=\sqrt{\frac{2}{\pi k_{\rho} \rho}} \exp \left[i k_{\rho} \rho-\frac{1}{2} i \pi\left(n+\frac{1}{2}\right)\right],
$$

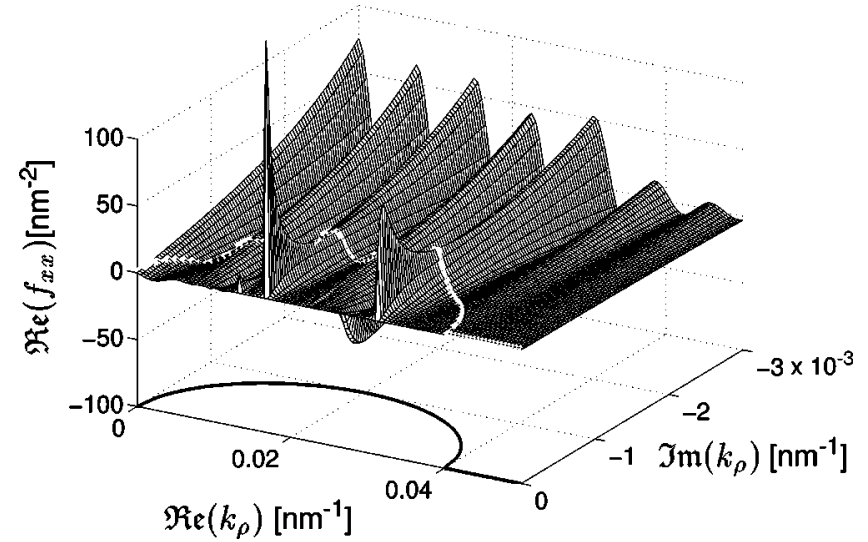

FIG. 4. Real part of the integrand $f_{x x}\left(k_{\rho}\right)$ [Eqs. (B1) and (B2)]. In this example, a nonmagnetic three-layer system with $\epsilon_{1}=1, \epsilon_{2}$ $=4$, and $\epsilon_{3}=2$ is used. The elliptical integration path used to avoid the singularities is shown on the integrand (white line) and on the projection plane.

we can deflect the integration path from the real axis to a path parallel to the imaginary axis: for the first term in Eq. (18) in the positive imaginary direction, for the second term in the negative imaginary direction (Fig. 3). Thus, for each $\left|k_{\rho} \rho\right|$ the Hankel function must only be calculated for the argument with the positive imaginary part. The exponential term in Eq. (19) ensures then a much faster convergence of the integrand. This is illustrated in Fig. 5, where we compare the integrand using Bessel functions along the real $k_{\rho}$ axis with that corresponding to Hankel functions parallel to the imaginary $k_{\rho}$ axis. Evidently, the integration using Hankel functions converges much faster.

From a practical point of view, we use the Gauss-Kronrod quadrature to evaluate the integrals along the deformed path [28]. This efficient algorithm can easily handle the oscillating behavior of the Bessel and Hankel functions for large $k_{\rho} \rho$. We are using the 15 -point Gauss-Kronrod quadrature and stop the integration when a relative accuracy of $10^{-9}$ is

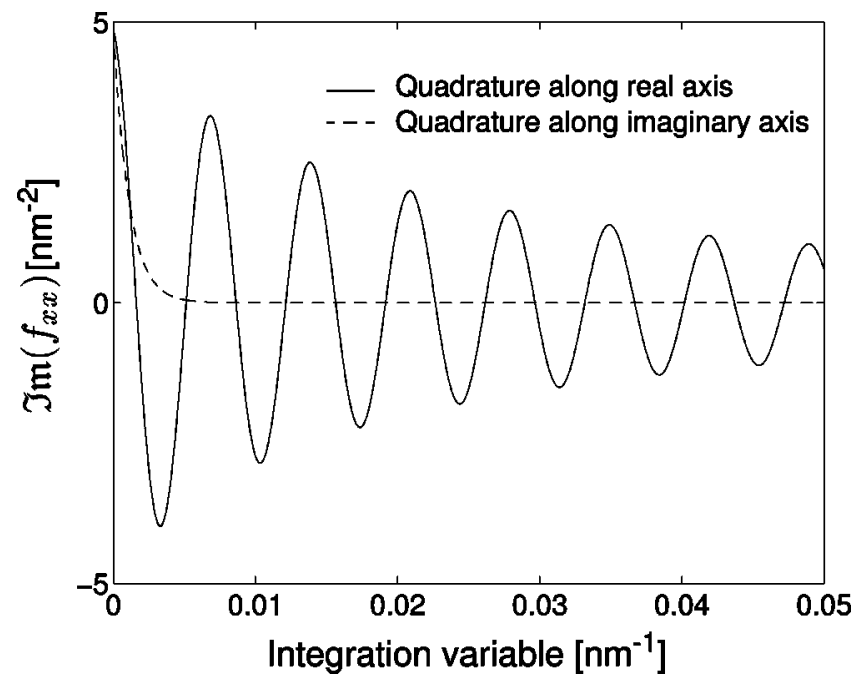

FIG. 5. Comparison of the imaginary part of the integrand $f_{x x}\left(k_{\rho}\right)$ along the real axis and the imaginary axis, respectively, using a formulation in terms of Bessel (solid line) and Hankel functions (dashed line), respectively. The system is identical to that used in Fig. 4. 


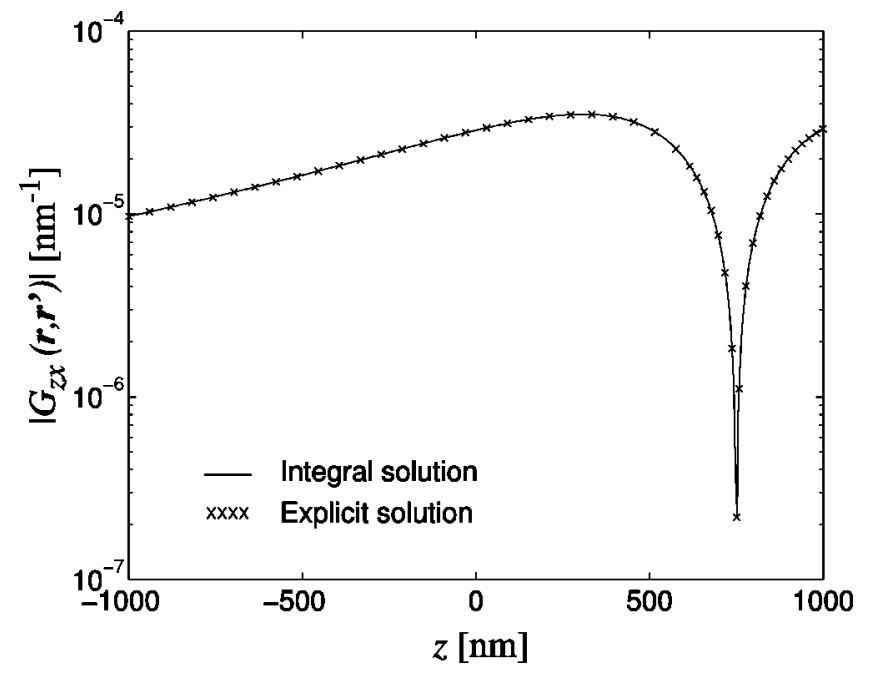

FIG. 6. Accuracy of the method: Comparison of the Green's tensor for a homogeneous medium obtained by numerical quadrature assuming four layers with identical permittivity (solid line) with that obtained from the explicit free-space solution [Eq. (3), crosses]. The $\left|G_{z x}\right|$ component is shown.

achieved. The application of a much more costly 64-point Gauss-Kronrod quadrature would roughly double the computation time without an appreciable gain in precision. Decreasing the desired relative accuracy to $10^{-6}$ typically speeds up the computation by a factor 1.5 .

A very useful trick to expedite the computation of the Green's tensor for a stratified medium is to integrate simultaneously the different components of $\mathbf{G}\left(\mathbf{r}, \mathbf{r}^{\prime}\right)$. However, we do not evaluate directly the individual components but rather choose a numerically more appropriate decomposition: The integrands in Eqs. (B1)-(B18) can be ordered in $s$ - and $p$-polarized terms with first- and second-order Bessel or Hankel functions. Thus, seven independent terms can be defined. We have observed that for a given $\left(\mathbf{r}, \mathbf{r}^{\prime}\right)$ pair these terms have a similar behavior in the $\mathbf{k}_{\rho}$ plane, which makes possible their simultaneous integration. In our practical implementation, we integrate in parallel 14 real functions that correspond to the real and imaginary parts of these independent contributions to the Green's tensor. As a measure for the Gauss-Kronrod quadrature (stop criterion), we simply use the sum of these 14 functions.

On our system (IBM PS/6000 7012-397, $160 \mathrm{MHz}$ ) the calculation of the complete Green's tensor for $1000\left(\mathbf{r}, \mathbf{r}^{\prime}\right)$ pairs takes for the example shown in Fig. 9 approximately $380 \mathrm{~s}$ of CPU time. Note, however, that this calculation time strongly depends on the relative position of $\mathbf{r}$ and $\mathbf{r}^{\prime}$.

To demonstrate the accuracy of our integration scheme, we first perform calculations for a system consisting of virtual layers, i.e., layers with identical permittivity and permeability. These results must be identical to the explicit solution given by the free-space Green's tensor of a homogeneous medium, Eq. (3). Figure 6 shows a comparison for a virtual four-layer medium. Obviously, the agreement between the two calculations is perfect.

\section{ILLUSTRATIVE EXAMPLES}

To illustrate the physical substance of our mathematical formalism, let us consider the most simple case of a nonmag- netic two-layer system $\epsilon_{1}, \epsilon_{2}$ separated by a single interface at $d=0$. Assuming that $\mathbf{r}^{\prime}$ lies within layer 1 and $\mathbf{r}$ within layer 2, the integrand $G_{z z}\left(k_{\rho} ; \rho, \phi\right)$ reduces to

$G_{z z}\left(k_{\rho} ; \mathbf{r}, \mathbf{r}^{\prime}\right)=\frac{k_{\rho}^{3}}{k_{2}^{2} k_{1 z}} J_{0}\left(k_{\rho} \rho\right) \mathcal{T}_{12}^{p} \exp \left[i\left(k_{1 z} z^{\prime}-k_{2 z} z\right)\right]$,

where $\mathcal{T}_{12}^{p}=1+\mathcal{F}_{12}^{p}=\epsilon_{2} k_{1 z} /\left(\epsilon_{2} k_{1 z}+\epsilon_{1} k_{2 z}\right)$ is the transmission coefficient of the surface.

As already mentioned, branch point singularities exist for $k_{\rho}= \pm k_{1}, \pm k_{2}$. By definition, for any $k_{\rho}$ lying on one of these branch cuts $k_{z}$ is pure real, forming a wave propagating in $z \rightarrow \pm \infty$ direction. These waves form a continuum of modes called radiation modes.

In addition, pole singularities appear when the denominator of $\mathcal{T}_{12}^{p}$ vanishes, i.e., for $k_{\rho}$ such that

$$
\epsilon_{2} \sqrt{k_{1}^{2}-k_{\rho}^{2}}+\epsilon_{1} \sqrt{k_{2}^{2}-k_{\rho}^{2}}=0 .
$$

The solutions of Eq. (21) are formally given by

$$
k_{\rho}= \pm \sqrt{\frac{\epsilon_{1} \epsilon_{2}}{\epsilon_{1}+\epsilon_{2}}} k_{0} \text {. }
$$

However, one has to verify if this solution is physically meaningful. If we choose $\epsilon_{1}$ and $\epsilon_{2}$ purely real and positive, obviously $k_{\rho}<k_{1}, k_{2}$. Hence, the square roots being also purely real and positive, Eq. (21) is not fulfilled. If we now assume that $\epsilon_{2}<0$ and $\epsilon_{1}<\left|\epsilon_{2}\right|$, a solution does exist. For such a plasma medium, the wave vectors in the $z$ direction $k_{1 z}$ and $k_{2 z}$ at the location of the pole $k_{\rho}$ become

$$
\begin{gathered}
k_{i z}=\sqrt{k_{i}^{2}-k_{\rho}^{2}}=\sqrt{\epsilon_{i}-\frac{\epsilon_{1} \epsilon_{2}}{\epsilon_{1}+\epsilon_{2}}} k_{0}=\sqrt{\frac{\epsilon_{i}^{2}}{\epsilon_{1}+\epsilon_{2}}} k_{0} \\
\text { for } i=1,2 .
\end{gathered}
$$

Refering to the discussion in Sec. III A, the sign of the square root in Eq. (23) has to be chosen such that the imaginary part is non-negative. Hence, $k_{1 z}$ and $k_{2 z}$ are pure imaginary and the field decays exponentially from the interface. The pole constitutes a surface-plasmon mode. Similarly, for an appropriate choice of the material properties other surface modes can be found by analyzing the pole locations [29].

In a three-layer structure, in addition to the branch point singularities at $k_{\rho}= \pm k_{1}, \pm k_{3}$, pole singularities associated with guided modes can occur. If $k_{\rho}^{2}>k_{1}^{2}$ and $k_{\rho}^{2}>k_{3}^{2}$, the wave vectors in the $z$ direction $k_{1 z}=\left(k_{1}^{2}-k_{\rho}^{2}\right)^{1 / 2}$ and $k_{3 z}$ $=\left(k_{3}^{2}-k_{\rho}^{2}\right)^{1 / 2}$ are imaginary and the field decreases exponentially in these outermost layers. Hence, $k_{\rho}$ corresponds to the modes guided by the slab. Their number, i.e., the number of poles, depends on the wavelength and the thickness of the intermediate layer [30].

As an illustration, Fig. 7 shows the $s$-polarized contribution of the integrand along the real axis for a symmetric planar $\mathrm{GaAs} / \mathrm{Al}_{0.15} \mathrm{Ga}_{0.85} \mathrm{As} / \mathrm{GaAs}$ waveguide structure at a $\lambda=1.5 \mu \mathrm{m}$ wavelength. According to a guided mode analysis, a similar structure with a $\Delta=0.5 \mu \mathrm{m}$ thick AlGaAs layer has one single $s$-polarized mode, located at $k_{\rho}$ $=14.6 \mu \mathrm{m}^{-1}$. For a thicker AlGaAs layer, $\Delta=2 \mu \mathrm{m}$, three $s$-polarized modes exist with, respectively, $k_{\rho}$ 


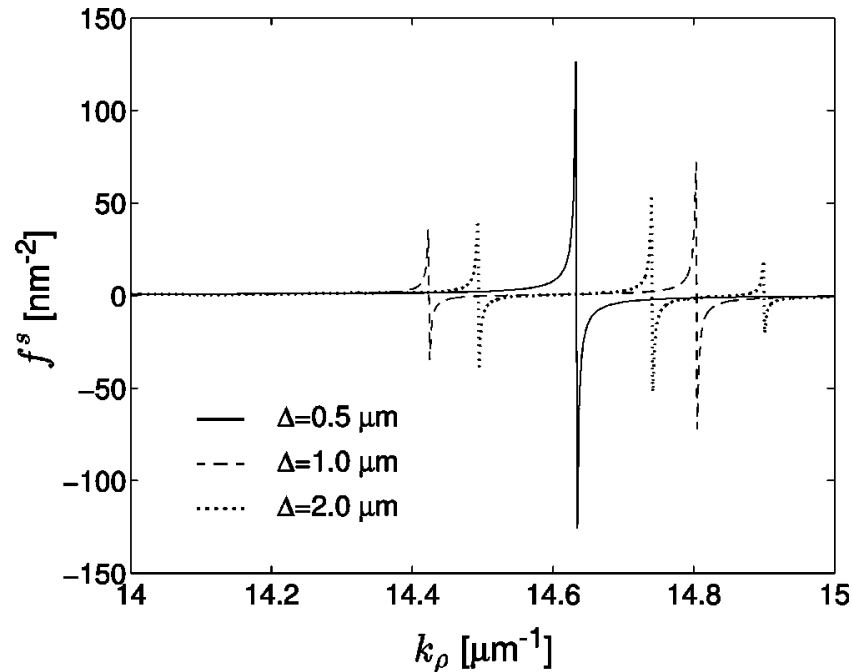

FIG. 7. Integrand ( $s$ polarization) for a symmetric $\mathrm{GaAs} / \mathrm{Al}_{0.15} \mathrm{Ga}_{0.85} \mathrm{As} / \mathrm{GaAs}$ planar waveguide, for three different widths $\Delta$ of the AlGaAs layer. The wavelength is $\lambda=1.5 \mu \mathrm{m}$.

$=14.9 \mu \mathrm{m}^{-1}, 14.7 \mu \mathrm{m}^{-1}$, and $14.5 \mu \mathrm{m}^{-1}$. In Fig. 7 we observe that the integrands' divergences coincide perfectly with these eigenmodes.

We can also investigate more complicated structures, such as a planar waveguide coupler: We consider a symmetric GaAs/AlGaAs/GaAs/AlGaAs/GaAs system with the two guiding $\mathrm{Al}_{0.15} \mathrm{Ga}_{0.85} \mathrm{As}$ layers having identical thicknesses $\Delta=0.5 \mu \mathrm{m}$. The $s$-polarized contribution of the integrand along the real axis is plotted in Fig. 8. For a thick separating GaAs layer, $\Delta^{\prime}=2 \mu \mathrm{m}$, the AlGaAs slabs are decoupled: only one $s$-polarized mode can be excited at $k_{\rho}$ $=14.6 \mu \mathrm{m}^{-1}$, as in the previous example. However, with decreasing separating layer thickness, the modes of the two waveguides can couple via their evanescent tails. The mode is split and the behavior of the structure develops towards that of a single-layer waveguide. For a thin separating GaAs layer, $\Delta^{\prime}=0.05 \mu \mathrm{m}$, the poles are located at $k_{\rho}$

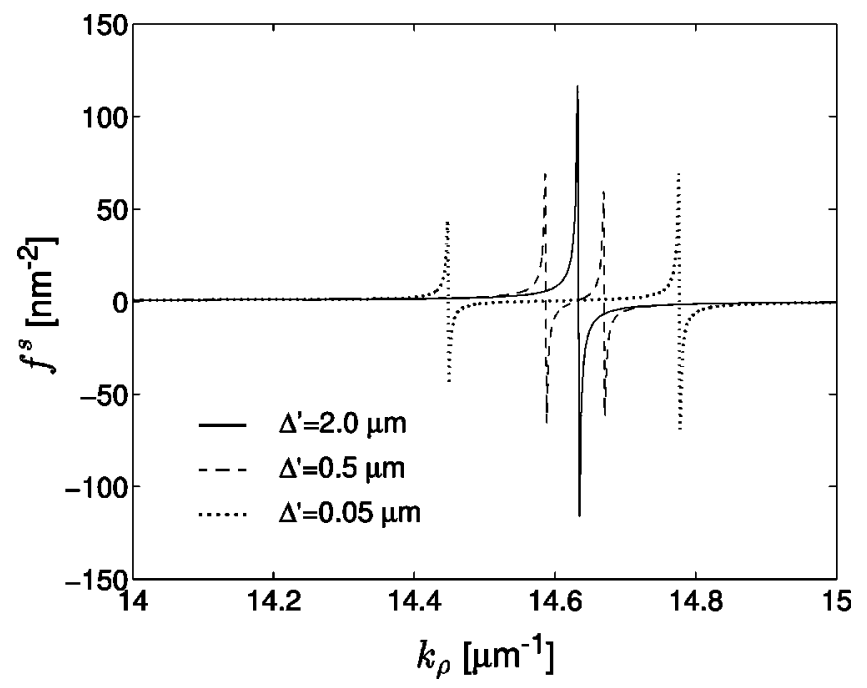

FIG. 8. Integrand ( $s$ polarization) for a GaAs/ $\mathrm{AlGaAs} / \mathrm{GaAs} / \mathrm{AlGaAs} / \mathrm{GaAs}$ multilayer structure, for three different widths $\Delta^{\prime}$ of the separating GaAs layer. The wavelength is $\lambda$ $=1.5 \mu \mathrm{m}$.

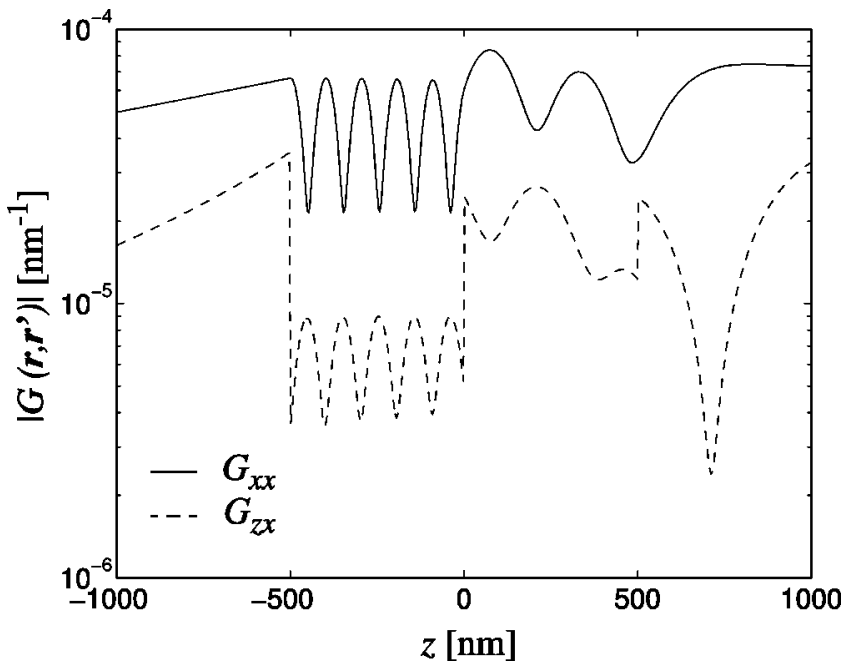

FIG. 9. $\left|G_{x x}\right|$ and $\left|G_{z x}\right|$ components of the Green's tensor for a four-layer structure with $\epsilon_{1}=1, \epsilon_{2}=2, \epsilon_{3}=10$, and $\epsilon_{4}=1$. The second layer extends from $z=0 \mathrm{~nm}$ to $z=-500 \mathrm{~nm}$, the third layer to $z=-1000 \mathrm{~nm}$. $z^{\prime}=750 \mathrm{~nm}$ is located in the top layer and held fixed.

$=14.8 \mu \mathrm{m}^{-1}$ and $14.4 \mu \mathrm{m}^{-1}$, which corresponds to the modes of a single-layer waveguide with $\Delta=0.5 \mu \mathrm{m}$ $+0.5 \mu \mathrm{m}=1 \mu \mathrm{m}$.

As the last example, we consider a four-layer medium with $\epsilon_{1}=1, \epsilon_{2}=2, \epsilon_{3}=10$, and $\epsilon_{4}=1$ at a $\lambda=633 \mathrm{~nm}$ wavelength. The thickness of each inner layer is $500 \mathrm{~nm}$. Two components of the Green's tensor, $G_{x x}\left(\mathbf{r}, \mathbf{r}^{\prime}\right)$ and $G_{z x}\left(\mathbf{r}, \mathbf{r}^{\prime}\right)$, are given as a function of the altitude $z$ of the observation point $\mathbf{r}=(\rho, \Phi, z)=(\lambda, \pi / 4, z)$, whereas the source point $\mathbf{r}^{\prime}=(0,0,750 \mathrm{~nm})$ is held constant in the top layer. Physically, these functions represent the $x$ and $z$ components of the electric field radiated by an $x$-oriented electric dipole. In the slab regions the dipole source excites standing waves with a period $\Lambda_{2}=224 \mathrm{~nm}$ and $\Lambda_{3}=100 \mathrm{~nm}$, which correspond to the half-effective wavelength in the according layer. (Note, however, that our source is a dipole, not a plane wave.) Figure 9 also illustrates the continuity properties across the interfaces. The tangential component of the electric field is continuous across all interfaces [Eq. (A1a)], whereas the normal component of the electric displacement is continuous [Eq. (A1b)]. Hence, $G_{z x}\left(\mathbf{r}, \mathbf{r}^{\prime}\right)$ jumps at the interfaces by a factor $\epsilon_{3} / \epsilon_{2}=5$ and $\epsilon_{3} / \epsilon_{4}=10$, respectively.

\section{SUMMARY}

We have presented a technique for the computation of the Green's tensor in stratified media. The computation details have been extensively discussed, which should make the practical implementation of this technique on a computer straightforward.

Several examples demonstrated the accuracy of this approach and illustrated the intrinsic physical properties of the Green's tensor in a stratified medium. This dyadic can also be used in conjunction with the Green's tensor technique for efficient scattering calculations in media composed of an arbitrary number of layers [16]. 


\section{ACKNOWLEDGMENTS}

The authors thank B. Michel for his support of the project and gratefully acknowledge the funding of the Swiss $\mathrm{Na}$ tional Science Foundation.

\section{APPENDIX A}

In this appendix we compute the amplitude coefficients $A_{l, \alpha \beta}^{s / p}$ and $B_{l, \alpha \beta}^{s / p}$, respectively, for $s / p$-polarized upgoing and downgoing waves in the different layers forming the structure [see Eqs. (10) and (B1)-(B18)]. These coefficients can be summarized in the matrices $\mathbf{A}_{l}^{s}, \mathbf{B}_{l}^{s}, \mathbf{A}_{l}^{p}$, and $\mathbf{B}_{l}^{p}$.

At each interface the Green's tensor must fulfill the continuity equations resulting from Maxwell's equations:

$$
\begin{gathered}
\hat{\mathbf{z}} \times\left(\mathbf{G}^{+}-\mathbf{G}^{-}\right)=\mathbf{0}, \\
\hat{\mathbf{z}} \cdot\left(\boldsymbol{\epsilon}^{+} \mathbf{G}^{+}-\boldsymbol{\epsilon}^{-} \mathbf{G}^{-}\right)=\mathbf{0},
\end{gathered}
$$

$$
\begin{gathered}
\hat{\mathbf{z}} \times \boldsymbol{\nabla} \times\left(\frac{\mathbf{G}^{+}}{\mu^{+}}-\frac{\mathbf{G}^{-}}{\mu^{-}}\right)=\mathbf{0}, \\
\hat{\mathbf{z}} \cdot \boldsymbol{\nabla} \times\left(\mathbf{G}^{+}-\mathbf{G}^{-}\right)=\mathbf{0},
\end{gathered}
$$

where $\mathbf{G}^{+}, \epsilon^{+}$, and $\mu^{+}$denote the quantities just above and $\mathbf{G}^{-}, \boldsymbol{\epsilon}^{-}$, and $\mu^{-}$just below the interface. Equations (A1a) and (A1b) are the boundary conditions for the electric field, whereas Eqs. (A1c) and (A1d) are the boundary conditions for the magnetic field.

Using Eqs. (A1a)-(A1d), we can deduce iteratively the amplitude ratios of upgoing and downgoing waves in each layer $l$. For that purpose, we consider separately the case when $\mathbf{r}$ is above $\mathbf{r}^{\prime}$ and that when it is below.

When $z>z^{\prime}$, we use the fact that no field is reflected from infinity $(z \rightarrow+\infty)$, so that $B_{1, \alpha \beta}=0$. Thus, we can iteratively determine the amplitude ratio of the downgoing/upgoing wave in each layer $l=2, \ldots, l^{\prime}$ using the relation

$$
\left(\frac{B_{l}}{A_{l}}\right)_{z>z^{\prime}}^{s / p}=\frac{\mathcal{F}_{l, l-1}^{s / p} \exp \left[i d_{l-1}\left(k_{l-1 z}+k_{l z}\right)\right]+\left(\frac{B_{l-1}}{A_{l-1}}\right)_{z>z^{\prime}}^{s / p} \exp \left[-i d_{l-1}\left(k_{l-1 z}-k_{l z}\right)\right]}{\exp \left[i d_{l-1}\left(k_{l-1 z}-k_{l z}\right)\right]+\mathcal{F}_{l, l-1}^{s / p}\left(\frac{B_{l-1}}{A_{l-1}}\right)_{z>z^{\prime}}^{s / p} \exp \left[-i d_{l-1}\left(k_{l-1 z}+k_{l z}\right)\right]} .
$$

In a similar way, for $z<z^{\prime}$, we use the fact $A_{N, \alpha \beta}=0$ (no field is reflected from $z \rightarrow-\infty$ ). Now we obtain for the amplitude ratio of the upgoing/downgoing wave in each layer $l=N-1, \ldots, l^{\prime}$,

$$
\left(\frac{A_{l}}{B_{l}}\right)_{z<z^{\prime}}^{s / p}=\frac{\mathcal{F}_{l, l+1}^{s / p} \exp \left[-i d_{l}\left(k_{l+1 z}+k_{l z}\right)\right]+\left(\frac{A_{l+1}}{B_{l+1}}\right)_{z<z^{\prime}}^{s / p} \exp \left[i d_{l}\left(k_{l+1 z}-k_{l z}\right)\right]}{\exp \left[-i d_{l}\left(k_{l+1 z}-k_{l z}\right)\right]+\mathcal{F}_{l, l+1}^{s / p}\left(\frac{A_{l+1}}{B_{l+1}}\right)_{z<z^{\prime}}^{s / p} \exp \left[i d_{l}\left(k_{l+1 z}+k_{l z}\right)\right]} .
$$

The coefficients $\mathcal{F}_{l, l-1}^{s / p}$ and $\mathcal{F}_{l, l+1}^{s / p}$ in Eqs. (A2a) and (A2b) are the Fresnel reflection coefficients:

$$
\begin{gathered}
\mathcal{F}_{l, l \pm 1}^{s}=\frac{\mu_{l \pm 1} k_{l z}-\mu_{l} k_{l \pm 1 z}}{\mu_{l \pm 1} k_{l z}+\mu_{l} k_{l \pm 1 z}}, \\
\mathcal{F}_{l, l \pm 1}^{p}=\frac{\epsilon_{l \pm 1} k_{l z}-\epsilon_{l} k_{l \pm 1 z}}{\epsilon_{l \pm 1} k_{l z}+\epsilon_{l} k_{l \pm 1 z}} .
\end{gathered}
$$

We dropped the $\alpha \beta$ indices in Eqs. (A2a) and (A2b) because the ratio is the same for each component.

In the layer $l^{\prime}$ the amplitudes can be explicitly calculated. According to Eq. (9), the total field in this region can be written as a superposition of the direct nonreflected wave and the reflected upgoing and downgoing waves:

$\mathcal{A}_{l^{\prime}, \alpha \beta}^{s / p} \exp \left(i k_{l^{\prime} z} z\right)+\mathcal{B}_{l^{\prime}, \alpha \beta}^{s / p} \exp \left(-i k_{l^{\prime} z} z\right)$

$$
\begin{aligned}
= & \mathcal{C}_{l^{\prime}, \alpha \beta}^{s / p} \exp \left(i k_{l^{\prime} z}\left|z-z^{\prime}\right|\right) \\
& +R_{l^{\prime}, \alpha \beta}^{s / p \uparrow} \exp \left(i k_{l^{\prime} z^{\prime}} z\right)+R_{l^{\prime}, \alpha \beta}^{s / p \downarrow} \exp \left(-i k_{l^{\prime} z} z\right),
\end{aligned}
$$

where $\mathcal{A}_{l^{\prime}, \alpha \beta}^{s / p}$ is the amplitude of the upgoing wave, $\mathcal{B}_{l^{\prime}, \alpha \beta}^{s / p}$ the amplitude of the downgoing wave, and $\mathcal{C}_{l^{\prime}, \alpha \beta}^{s / p}$ the amplitude of the direct wave [see Eq. (9)]:

$$
\begin{aligned}
& \mathcal{C}_{l^{\prime}, \alpha \beta}^{s}=\hat{l}_{\alpha}\left(k_{l^{\prime} z}\right) \hat{l}_{\beta}\left(k_{l^{\prime} z}\right), \\
& \mathcal{C}_{l^{\prime}, \alpha \beta}^{p}=\hat{m}_{\alpha}\left(k_{l^{\prime} z}\right) \hat{m}_{\beta}\left(k_{l^{\prime} z}\right) .
\end{aligned}
$$

Evaluating the products in Eqs. (A5a) and (A5b) using Eqs. (6) and (7) shows immediately that $\mathcal{C}_{l^{\prime}, x z}^{p}, \mathcal{C}_{l^{\prime}, y z}^{p}, \mathcal{C}_{l^{\prime}, z x}^{p}$, and $\mathcal{C}_{l^{\prime}, z y}^{p}$ are odd about $z=z^{\prime}$, whereas all the other amplitudes are even about $z=z^{\prime}$. Note that the amplitudes $\mathcal{A}_{l^{\prime}, \alpha \beta}^{s / p}$ and $\mathcal{B}_{l^{\prime}, \alpha \beta}^{s / p}$ are not identical to the coefficients $A_{l^{\prime}, \alpha \beta}^{s / p}$ and $B_{l^{\prime}, \alpha \beta}^{s / p}$ used above. However, they can be connected by comparison 
with the integrands given in Appendix B. For example, for the $p$-polarized part of the $x x$ component, they are coupled via

$$
\begin{aligned}
\mathcal{A}_{l^{\prime}, x x}^{p} & =R_{l^{\prime}, x x}^{p \uparrow}+\Theta\left(z-z^{\prime}\right) \mathcal{C}_{l^{\prime}, x x}^{p} \exp \left(-i k_{l^{\prime} z} z^{\prime}\right) \\
& = \pm \mathcal{C}_{l^{\prime}, x x}^{p} A_{l^{\prime}, x x}^{p}, \\
\mathcal{B}_{l^{\prime}, x x}^{p} & =R_{l^{\prime}, x x}^{p \downarrow}+\Theta\left(z^{\prime}-z\right) \mathcal{C}_{l^{\prime}, x x}^{p} \exp \left(i k_{l^{\prime} z^{\prime}} z^{\prime}\right) \\
& =\mp \mathcal{C}_{l^{\prime}, x x}^{p} B_{l^{\prime}, x x}^{p},
\end{aligned}
$$

where the upper sign refers to $z>z^{\prime}$ and the lower sign to $z<z^{\prime}$.

Using Eq. (A4), the amplitude ratio of the downgoing/ upgoing wave reads for $z>z^{\prime}$

$$
\left(\frac{\mathcal{B}_{l^{\prime}, \alpha \beta}}{\mathcal{A}_{l^{\prime}, \alpha \beta}}\right)_{z>z^{\prime}}^{s / p}=\frac{R_{l^{\prime}, \alpha \beta}^{s / p \downarrow}}{\mathcal{C}_{l^{\prime}, \alpha \beta}^{s / p} \exp \left(-i k_{l^{\prime} z} z^{\prime}\right)+R_{l^{\prime}, \alpha \beta}^{s / p \uparrow}},
$$

and the amplitude ratio of the upgoing/downgoing wave becomes for $z<z^{\prime}$

$$
\left(\frac{\mathcal{A}_{l^{\prime}, \alpha \beta}}{\mathcal{B}_{l^{\prime}, \alpha \beta}}\right)_{z<z^{\prime}}^{s / p}=\frac{R_{l^{\prime}, \alpha \beta}^{s / p \uparrow}}{\mathcal{C}_{l^{\prime}, \alpha \beta}^{s / p} \exp \left(i k_{l^{\prime} z} z^{\prime}\right)+R_{l^{\prime}, \alpha \beta}^{s / p \downarrow}} .
$$

Solving Eqs. (A7a) and (A7b) for $R_{l^{\prime}, \alpha \beta}^{s / p \downarrow}$ and $R_{l^{\prime}, \alpha \beta}^{s / p \uparrow}$ and substituting the result into the equations formed in analogy to Eqs. (A6a) and (A6b) gives the components of $\mathbf{A}_{l^{\prime}}^{s / p}$ and $\mathbf{B}_{l^{\prime}}^{s / p}$. One only has to replace the amplitude ratios formed by $\mathcal{A}_{l^{\prime}, \alpha \beta}^{s / p}$ and $\mathcal{B}_{l^{\prime}, \alpha \beta}^{s / p}$ with the ratios of the corresponding coefficients $A_{l^{\prime}, \alpha \beta}^{s / p}$ and $B_{l^{\prime}, \alpha \beta}^{s / p}$, keeping in mind the differences between $z>z^{\prime}$ and $z<z^{\prime}$. It turns out that for $p$ polarization two cases must be distinguished. For $\beta \neq z$ the solution reads

$$
\begin{aligned}
A_{l^{\prime}, \alpha \beta}^{p}= & \pm\left(\frac{A_{l^{\prime}}}{B_{l^{\prime}}}\right)_{z<z^{\prime}}^{p}\left[1-\left(\frac{B_{l^{\prime}}}{A_{l^{\prime}}}\right)_{z>z^{\prime}}^{p}\left(\frac{A_{l^{\prime}}}{B_{l^{\prime}}}\right)_{z<z^{\prime}}^{p}\right]^{-1} \\
\times & {\left[\left(\frac{B_{l^{\prime}}}{A_{l^{\prime}}}\right)_{z>z^{\prime}}^{p} \exp \left(-i k_{l^{\prime} z} z^{\prime}\right)-\exp \left(i k_{l^{\prime}} z^{\prime}\right)\right] } \\
\pm & \Theta\left(z-z^{\prime}\right) \exp \left(-i k_{l^{\prime} z} z^{\prime}\right), \\
B_{l^{\prime}, \alpha \beta}^{p}= & \mp\left(\frac{B_{l^{\prime}}}{A_{l^{\prime}}}\right)_{z>z^{\prime}}^{p}\left[1-\left(\frac{B_{l^{\prime}}}{A_{l^{\prime}}}\right)_{z>z^{\prime}}^{p}\left(\frac{A_{l^{\prime}}}{B_{l^{\prime}}}\right)_{z<z^{\prime}}^{p}\right]^{-1} \\
& \times\left[\left(\frac{A_{l^{\prime}}}{B_{l^{\prime}}}\right)_{z<z^{\prime}}^{p} \exp \left(i k_{l^{\prime} z} z^{\prime}\right)-\exp \left(-i k_{l^{\prime} z} z^{\prime}\right)\right] \\
& \mp \Theta\left(z^{\prime}-z\right) \exp \left(i k_{l^{\prime}} z^{\prime}\right),
\end{aligned}
$$

whereas for $\beta=z, A_{l^{\prime}, \alpha \beta}^{p}$ and $B_{l^{\prime}, \alpha \beta}^{p}$ are given by

$$
\begin{aligned}
A_{l^{\prime}, \alpha z}^{p}= & \left(\frac{A_{l^{\prime}}}{B_{l^{\prime}}}\right)_{z<z^{\prime}}^{p}\left[1-\left(\frac{B_{l^{\prime}}}{A_{l^{\prime}}}\right)_{z>z^{\prime}}^{p}\left(\frac{A_{l^{\prime}}}{B_{l^{\prime}}}\right)_{z<z^{\prime}}^{p}\right]^{-1} \\
& \times\left[\left(\frac{B_{l^{\prime}}}{A_{l^{\prime}}}\right)_{z>z^{\prime}}^{p} \exp \left(-i k_{l^{\prime} z^{\prime}} z^{\prime}\right)+\exp \left(i k_{l^{\prime} z} z^{\prime}\right)\right] \\
& +\Theta\left(z-z^{\prime}\right) \exp \left(-i k_{l^{\prime} z} z^{\prime}\right), \\
B_{l^{\prime}, \alpha z}^{p}= & \left(\frac{B_{l^{\prime}}}{A_{l^{\prime}}}\right)_{z>z^{\prime}}^{p}\left[1-\left(\frac{B_{l^{\prime}}}{A_{l^{\prime}}}\right)_{z>z^{\prime}}^{p}\left(\frac{A_{l^{\prime}}}{B_{l^{\prime}}}\right)_{z<z^{\prime}}^{p}\right]^{-1} \\
& \times\left[\left(\frac{A_{l^{\prime}}}{B_{l^{\prime}}}\right)_{z<z^{\prime}}^{p} \exp \left(i k_{l^{\prime} z} z^{\prime}\right)+\exp \left(-i k_{l^{\prime} z} z^{\prime}\right)\right] \\
& +\Theta\left(z^{\prime}-z\right) \exp \left(i k_{l^{\prime} z} z^{\prime}\right) .
\end{aligned}
$$

Physically, this difference is due to the fact that the components with $\beta=z$ represent the field of a vertical dipole whereas the other components come from a horizontal dipole.

Since an $s$-polarized wave is only generated by a horizontal dipole, such a distinction does not occur:

$$
\begin{aligned}
A_{l^{\prime}, \alpha \beta}^{s}= & \left(\frac{A_{l^{\prime}}}{B_{l^{\prime}}}\right)_{z<z^{\prime}}^{s}\left[1-\left(\frac{B_{l^{\prime}}}{A_{l^{\prime}}}\right)_{z>z^{\prime}}^{s}\left(\frac{A_{l^{\prime}}}{B_{l^{\prime}}}\right)_{z<z^{\prime}}^{s}\right]^{-1} \\
& \times\left[\left(\frac{B_{l^{\prime}}}{A_{l^{\prime}}}\right)_{z>z^{\prime}}^{s} \exp \left(-i k_{l^{\prime} z^{\prime}} z^{\prime}\right)+\exp \left(i k_{l^{\prime} z} z^{\prime}\right)\right] \\
& +\Theta\left(z-z^{\prime}\right) \exp \left(-i k_{l^{\prime} z} z^{\prime}\right), \\
B_{l^{\prime}, \alpha \beta}^{s}= & \left(\frac{B_{l^{\prime}}}{A_{l^{\prime}}}\right)_{z>z^{\prime}}^{s}\left[1-\left(\frac{B_{l^{\prime}}}{A_{l^{\prime}}}\right)_{z>z^{\prime}}^{s}\left(\frac{A_{l^{\prime}}}{B_{l^{\prime}}}\right)_{z<z^{\prime}}^{s}\right]^{-1} \\
& \times\left[\left(\frac{A_{l^{\prime}}}{B_{l^{\prime}}}\right)_{z<z^{\prime}}^{s} \exp \left(i k_{l^{\prime}} z^{\prime}\right)+\exp \left(-i k_{l^{\prime}} z^{\prime}\right)\right] \\
& +\Theta\left(z^{\prime}-z\right) \exp \left(i k_{l^{\prime}} z^{\prime}\right) .
\end{aligned}
$$

In this case, $A_{l^{\prime}, \alpha \beta}^{s}$ and $B_{l^{\prime}, \alpha \beta}^{s}$ are only defined for $\alpha=x, y$ and $\beta=x, y$, whereas the five other components are set to zero.

In Eqs. (A8) and (A9) the amplitude coefficients in layer $l^{\prime}$ are expressed in terms of amplitude ratios which we have already calculated in Eqs. (A2b) and (A2a). Since the amplitude coefficients of the upgoing and downgoing waves in the layer $l$ are iteratively connected to the amplitude coefficients in $l^{\prime}$ via the boundary conditions (A1), we can explicitly calculate the components of $\mathbf{A}_{l}^{s / p}$ and $\mathbf{B}_{l}^{s / p}$, keeping in mind that the boundary conditions are different for the $x, y$, and for the $z$ components (note, however, that the $s$-polarized wave has no $z$ component). This relationship can be formulated using propagation matrices [31] with different factors $\gamma$. For $z>z^{\prime}$ we have 


$$
\left(\begin{array}{l}
A_{l, \alpha \beta}^{s / p} \\
B_{l, \alpha \beta}^{s / p}
\end{array}\right)=\gamma_{z>z^{\prime}}^{s / p}\left(\begin{array}{cc}
\exp \left[i d_{l}\left(k_{l+1 z}-k_{l z}\right)\right] & \mathcal{F}_{l, l+1}^{s / p} \exp \left[-i d_{l}\left(k_{l+1 z}+k_{l z}\right)\right] \\
\mathcal{F}_{l, l+1}^{s / p} \exp \left[i d_{l}\left(k_{l+1 z}+k_{l z}\right)\right] & \exp \left[-i d_{l}\left(k_{l+1 z}-k_{l z}\right)\right]
\end{array}\right)\left(\begin{array}{c}
A_{l+1, \alpha \beta}^{s / p} \\
B_{l+1, \alpha \beta}^{s / p}
\end{array}\right),
$$

where

$$
\begin{gathered}
\gamma_{z>z^{\prime}}^{s}=\frac{1}{2} \frac{\mu_{l+1} k_{l z}+\mu_{l} k_{l+1 z}}{\mu_{l+1} k_{l+1 z}} \text { for } \alpha=x, y, \beta=x, y, \\
\gamma_{z>z^{\prime}}^{p}= \begin{cases}\frac{1}{2} \frac{\mu_{l}}{\mu_{l+1}} \frac{k_{l+1 z}}{k_{l z}} \frac{\epsilon_{l+1} k_{l z}+\epsilon_{l} k_{l+1 z}}{\epsilon_{l+1} k_{l+1 z}} & \text { for } \alpha=x, y, \beta=x, y, z, \\
\frac{1}{2} \frac{\mu_{l}}{\mu_{l+1}} \frac{\epsilon_{l+1} k_{l z}+\epsilon_{l} k_{l+1 z}}{\epsilon_{l+1} k_{l+1 z}} & \text { for } \alpha=z, \beta=x, y, z .\end{cases}
\end{gathered}
$$

Similarly, for $z<z^{\prime}$ we obtain the following expressions:

$$
\left(\begin{array}{c}
A_{l, \alpha \beta}^{s / p} \\
B_{l, \alpha \beta}^{s / p}
\end{array}\right)=\gamma_{z<z^{\prime}}^{s / p}\left(\begin{array}{cc}
\exp \left[i d_{l-1}\left(k_{l-1 z}-k_{l z}\right)\right] & \mathcal{F}_{l, l-1}^{s / p} \exp \left[-i d_{l-1}\left(k_{l-1 z}+k_{l z}\right)\right] \\
\mathcal{F}_{l, l-1}^{s / p} \exp \left[i d_{l-1}\left(k_{l-1 z}+k_{l z}\right)\right] & \exp \left[-i d_{l-1}\left(k_{l-1 z}-k_{l z}\right)\right]
\end{array}\right)\left(\begin{array}{c}
A_{l-1, \alpha \beta}^{s / p} \\
B_{l-1, \alpha \beta}^{s / p}
\end{array}\right),
$$

where

$$
\begin{gathered}
\gamma_{z<z^{\prime}}^{s}=\frac{1}{2} \frac{\mu_{l-1} k_{l z}+\mu_{l} k_{l-1 z}}{\mu_{l-1} k_{l-1 z}} \text { for } \alpha=x, y, \beta=x, y, \\
\gamma_{z<z^{\prime}}^{p}= \begin{cases}\frac{1}{2} \frac{\mu_{l}}{\mu_{l-1}} \frac{k_{l-1 z}}{k_{l z}} \frac{\epsilon_{l-1} k_{l z}+\epsilon_{l} k_{l-1 z}}{\epsilon_{l-1} k_{l-1 z}} & \text { for } \alpha=x, y, \beta=x, y, z, \\
\frac{1}{2} \frac{\mu_{l}}{\mu_{l-1}} \frac{\epsilon_{l-1} k_{l z}+\epsilon_{l} k_{l-1 z}}{\epsilon_{l-1} k_{l-1 z}} & \text { for } \alpha=z, \beta=x, y, z .\end{cases}
\end{gathered}
$$

Like this, we obtain the amplitude of $s$ - and $p$-polarized upgoing/downgoing waves in each layer.

\section{APPENDIX B}

Using $\hat{\mathbf{l}}\left(-k_{l z}\right)=\hat{\mathbf{l}}\left(k_{l z}\right)$ and $\hat{\mathbf{m}}\left(-k_{l z}\right)=\left(-\hat{m}_{x}\left(k_{l z}\right),-\hat{m}_{y}\left(k_{l z}\right), \hat{m}_{z}\left(k_{l z}\right)\right)$ the components of the integrand for the Green's tensor (10) read, after evaluation of the angular integral,

$$
\begin{aligned}
& f_{x x}^{s}\left(k_{\rho} ; \rho, \phi\right)=\frac{1}{k_{l z}}\left(k_{\rho} J_{0}\left(k_{\rho} \rho\right) \sin ^{2} \phi+\frac{1}{\rho} J_{1}\left(k_{\rho} \rho\right) \cos 2 \phi\right)\left[A_{l}^{s} \exp \left(i k_{l z} z\right)+B_{l}^{s} \exp \left(-i k_{l z} z\right)\right], \\
& f_{x x}^{p}\left(k_{\rho} ; \rho, \phi\right)= \pm \frac{k_{l z}}{k_{l}^{2}}\left(k_{\rho} J_{0}\left(k_{\rho} \rho\right) \cos ^{2} \phi-\frac{1}{\rho} J_{1}\left(k_{\rho} \rho\right) \cos 2 \phi\right)\left[A_{l, x x}^{p} \exp \left(i k_{l z} z\right)-B_{l, x x}^{p} \exp \left(-i k_{l z} z\right)\right], \\
& f_{x y}^{s}\left(k_{\rho} ; \rho, \phi\right)=\frac{1}{k_{l z}}\left(-k_{\rho} J_{0}\left(k_{\rho} \rho\right)+\frac{2}{\rho} J_{1}\left(k_{\rho} \rho\right)\right) \sin \phi \cos \phi\left[A_{l}^{s} \exp \left(i k_{l z} z\right)+B_{l}^{s} \exp \left(-i k_{l z} z\right)\right], \\
& f_{x y}^{p}\left(k_{\rho} ; \rho, \phi\right)= \pm \frac{k_{l z}}{k_{l}^{2}}\left(k_{\rho} J_{0}\left(k_{\rho} \rho\right)-\frac{2}{\rho} J_{1}\left(k_{\rho} \rho\right)\right) \sin \phi \cos \phi\left[A_{l, x y}^{p} \exp \left(i k_{l z} z\right)-B_{l, x y}^{p} \exp \left(-i k_{l z} z\right)\right], \\
& f_{x z}^{s}\left(k_{\rho} ; \rho, \phi\right)=0, \\
& f_{x z}^{p}\left(k_{\rho} ; \rho, \phi\right)=-\frac{i k_{\rho}^{2}}{k_{l}^{2}} J_{1}\left(k_{\rho} \rho\right) \cos \phi\left[A_{l, x z}^{p} \exp \left(i k_{l z} z\right)-B_{l, x z}^{p} \exp \left(-i k_{l z} z\right)\right], \\
& f_{y x}^{s}\left(k_{\rho} ; \rho, \phi\right)=f_{x y}^{s}, \\
& f_{y x}^{p}\left(k_{\rho} ; \rho, \phi\right)=f_{x y}^{p},
\end{aligned}
$$




$$
\begin{aligned}
& f_{y y}^{s}\left(k_{\rho} ; \rho, \phi\right)=\frac{1}{k_{l z}}\left(k_{\rho} J_{0}\left(k_{\rho} \rho\right) \cos ^{2} \phi-\frac{1}{\rho} J_{1}\left(k_{\rho} \rho\right) \cos 2 \phi\right)\left[A_{l}^{s} \exp \left(i k_{l z} z\right)+B_{l}^{s} \exp \left(-i k_{l z} z\right)\right], \\
& f_{y y}^{p}\left(k_{\rho} ; \rho, \phi\right)= \pm \frac{k_{l z}}{k_{l}^{2}}\left(k_{\rho} J_{0}\left(k_{\rho} \rho\right) \sin ^{2} \phi+\frac{1}{\rho} J_{1}\left(k_{\rho} \rho\right) \cos 2 \phi\right)\left[A_{l, y y}^{p} \exp \left(i k_{l z} z\right)-B_{l, y y}^{p} \exp \left(-i k_{l z} z\right)\right], \\
& f_{y z}^{s}\left(k_{\rho} ; \rho, \phi\right)=0, \\
& f_{y z}^{p}\left(k_{\rho} ; \rho, \phi\right)=-\frac{i k_{\rho}^{2}}{k_{l}^{2}} J_{1}\left(k_{\rho} \rho\right) \sin \phi\left[A_{l, y z}^{p} \exp \left(i k_{l z} z\right)-B_{l, y z}^{p} \exp \left(-i k_{l z} z\right)\right], \\
& f_{z x}^{s}\left(k_{\rho} ; \rho, \phi\right)=0, \\
& f_{z x}^{p}\left(k_{\rho} ; \rho, \phi\right)=\mp \frac{i k_{\rho}^{2}}{k_{l}^{2}} J_{1}\left(k_{\rho} \rho\right) \cos \phi\left[A_{l, z x}^{p} \exp \left(i k_{l z} z\right)+B_{l, z x}^{p} \exp \left(-i k_{l z} z\right)\right], \\
& f_{z y}^{s}\left(k_{\rho} ; \rho, \phi\right)=0, \\
& f_{z y}^{p}\left(k_{\rho} ; \rho, \phi\right)=\mp \frac{i k_{\rho}^{2}}{k_{l}^{2}} J_{1}\left(k_{\rho} \rho\right) \sin \phi\left[A_{l, z y}^{p} \exp \left(i k_{l z} z\right)+B_{l, z y}^{p} \exp \left(-i k_{l z} z\right)\right], \\
& f_{z z}^{s}\left(k_{\rho} ; \rho, \phi\right)=0, \\
& f_{z z}^{p}\left(k_{\rho} ; \rho, \phi\right)=\frac{k_{\rho}^{3}}{k_{l}^{2} k_{l z}} J_{0}\left(k_{\rho} \rho\right)\left[A_{l, z z}^{p} \exp \left(i k_{l z} z\right)+B_{l, z z}^{p} \exp \left(-i k_{l z} z\right)\right] .
\end{aligned}
$$

The upper sign in these equations refers to $z>z^{\prime}$ and the lower sign to $z<z^{\prime}$. As mentioned in Appendix A [Eqs. (A9), (A11a), and (A13a)], all nonvanishing amplitude coefficients of $s$-polarized waves are equal, as denoted by the scalar $A_{l}^{s}$ and $B_{l}^{s}$. Note further that $A_{l, \alpha \beta}^{s / p}$ and $B_{l, \alpha \beta}^{s / p}$ are also functions of $k_{l z}$ and hence, because of $k_{l z}=\left(k_{l}^{2}-k_{\rho}^{2}\right)^{1 / 2}$, functions of $k_{\rho}$.

For $\rho=0$, some of the integrands are no longer defined because the denominator $\rho$ and the numerator $J_{1}\left(k_{\rho} \rho\right)$ van- ish. Furthermore, $\phi$ is not defined for $\rho=0$. However, explicit evaluation of Eq. (10) for $\rho=0$ shows that the upper formulas are still valid if we take their value for $\phi=0$ and make use of the limit

$$
\lim _{\rho \rightarrow 0} \frac{J_{1}\left(k_{\rho} \rho\right)}{\rho}=\frac{1}{2} k_{\rho} .
$$

[1] P.M. Morse and H. Feshbach, Methods of Theoretical Physics (McGraw-Hill, New York, 1953).

[2] C.-T. Tai, Dyadic Green Function in Electromagnetic Theory (IEEE Press, New York, 1994).

[3] A. Sommerfeld, Ann. Phys. (Leipzig) 28, 665 (1909).

[4] A. Baños, Dipole Radiation in the Presence of a Conducting Half-Space (Pergamon Press, Oxford, 1966).

[5] J.R. Mosig and F.E. Gardiol, IEE Proc. Pt.H: Microwaves. Opt. Antennas 130, 175 (1983).

[6] J.R. Wait, Electromagnetic Waves in Stratified Media (IEEE Press, New York, 1996).

[7] P. Gay-Balmaz and J.R. Mosig, Int. J. Microwave and Millimeter wave CAE 3, 330 (1997).

[8] G.S. Agarwal, Phys. Rev. A 11, 253 (1975).
[9] H. Metiu, Prog. Surf. Sci. 17, 153 (1984).

[10] G.W. Ford and W.H. Weber, Phys. Rep. 113, 195 (1984).

[11] F. Wijnands et al., Opt. Quantum Electron. 29, 199 (1997).

[12] A. Dereux, J.P. Vigneron, P. Lambin, and A.A. Lucas, Superlattices Microstruct. 3, 547 (1987).

[13] J. van Kranendonk and J. E. Sipe, in Progress in Optics, edited by E. Wolf (Elsevier, New York, 1977), Vol. 15.

[14] B.T. Draine and P.J. Flatau, J. Opt. Soc. Am. A 11, 1491 (1994).

[15] P.J. Flatau, Opt. Lett. 22, 1205 (1997).

[16] O.J.F. Martin and N.B. Piller, Phys. Rev. E 58, 3909 (1998).

[17] A.A. Maradudin and D.L. Mills, Phys. Rev. B 11, 1392 (1975).

[18] J.E. Sipe, J. Opt. Soc. Am. B 4, 481 (1987).

[19] M.A. Taubenblatt and T.K. Tran, J. Opt. Soc. Am. A 10, 912 
(1993).

[20] C. Girard, A. Dereux, O.J.F. Martin, and M. Devel, Phys. Rev. B 52, 2889 (1995).

[21] O.J.F. Martin, J. Microsc. 194, 235 (1999).

[22] J. A. Kong, Electromagnetic Wave Theory (Wiley, New York, 1986).

[23] Handbook of Mathematical Functions, edited by M. Abramowitz and I.A. Stegun (Dover, New York, 1972).

[24] L.B. Felsen and N. Marcuvitz, Radiation and Scattering of Waves (IEEE Press, New York, 1994).

[25] W.C. Chew, Waves and Fields in Inhomogeneous Media (IEEE Press, New York, 1995).
[26] R.G. Hunsperger, Integrated Optics: Theory and Technology, 3rd ed. (Springer, Berlin, 1991).

[27] L. Barlatey, J.R. Mosig, and T. Sphicopoulos, IEEE Trans. Antennas Propag. 38, 608 (1990).

[28] W. Gautschi, in Numerical Methods and Approximation Theory III, edited by G.V. Milovanović (Univ. Niš, Niš, 1988), pp. 39-66.

[29] A.D. Boardman, Electromagnetic Surface Modes (Wiley, New York, 1982).

[30] D. Marcuse, Theory of Dielectric Optical Waveguides (Academic Press, San Diego, 1974).

[31] J.A. Kong, Geophysics 37, 985 (1972). 